\title{
Simulation study on a DC-drive Electroosmotic Micromixer
}

\author{
Chenxiao Meng, Hanjun Li, Xiaoying Tang, Yuanqing $X u^{a, *}$ \\ School of Life Science, Beijing Institute of Technology, Beijing 100081, China \\ abitxyq@bit.edu.cn
}

Keywords: electroosmotic flow, zeta potential distribution, micromixer, DC-drive.

\begin{abstract}
In this paper, a type of DC-drive electroosmotic micro-mixer is proposed by numerical simulation. The model was built and optimized with multi-field coupling technique in finite element formation, in which the mixing efficiency was controlled by changing the Zeta potential distribution of the wall to make it under different driving voltage. In conclusion: (1) Significantly different characters can be observed by different zeta distribution settings; (2) Intensity of mixing can be improved by forming vortex with adequate size in the interface where the solute diffuse; (3) Compared with alternating current, the mixture in channel forced by direct current can get a more stable mixing performance.
\end{abstract}

\section{Introduction}

With the rapid expansion of microfabrication technology, MEMS is undergoing a great development. Microfluidic chip, as the good example of MEMS, has many advantages such as controllable fluid flow, less sample consumptions, high speed of analysis, which is playing a more important role in the field of chemistry, biology, engineering and physics [1]. The effective and efficient mixture of fluid is the key point in microfluidics, which is widely used in some research areas such as medical tests, chemical reactions [2]. However, fluid flows under small Reynold numbers in microns, which belongs to laminar state. In this situation, different solution can only be mixed by spreading and extending, which requires more spaces, longer period, and it is adverse for optimizing the design of microfluidic chip and improving its efficiency. Therefore, improvement on the form of mixture should be made. At present, there are two main designs: active and inactive. The efficiency of inactive micromixer is mainly improved by changing the shape of channels for increasing contact areas such as the T-type [3], L- type [4] and herringbone micromixer, those of which still have some defects such as complex structure, difficult to control. The active improve its efficiency mainly by affecting the laminar flow with the outside aid, which can often be seen in gas, magnetism, sound, electric heat and so on. Thereinto, electroosmosis is gaining great attention $[5,6]$, compared to other active mixer by its advantage in integration and easier way to improve the mix efficiency. As a result, electroosmotic micromixers were designed, which is proved to improve the chaotic mixing with the change of electroosmotic flow by controlling alternating voltage. Zhou $\mathrm{T}$ [7] and Maadi M [8] modify the shape of mix area and electrode location, thus improving the mix efficiency. Chen L [9] improve the design by adding ear chambers on both sides of the channel and put forward using low voltage direct electroosmotic pumps to make chaotic flow for increasing the complexity to speed up the mixing process.

The design of Electroosmotic micromixer has gained much attention and made a big progress in theoretical and experimental research, while there are some problems to be solved such as bubbles produced under high voltage will influence the mix process, heating problems. Based the research, nine models were designed. The test shows that the mix efficiency could be improved by improving the wall structure combined with Zeta potential distribution settings. 


\section{The Channel Structure and Electrode Design of the Micromixer}

\subsection{Channel structure and wall Zeta potential design}

Three types are illustrated below with different Zeta potential distribution. As is shown in No.1 diagram, red line represents Zeta potential value is positive, Blue line represents Zeta potential value is negative. Different Zeta potential distribution can be realized by different settings of coating material in the wall [10].

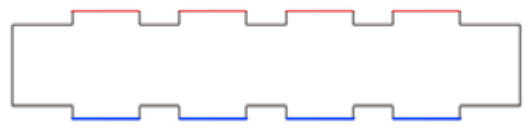

$\mathrm{A}_{1}$

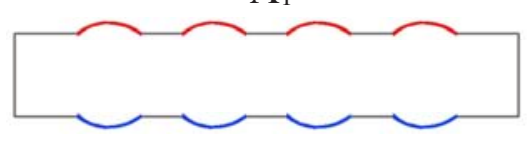

$\mathrm{B}_{1}$

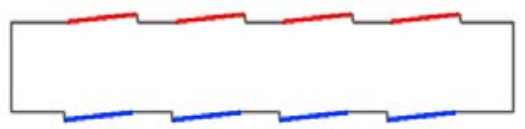

$\mathrm{C}_{1}$

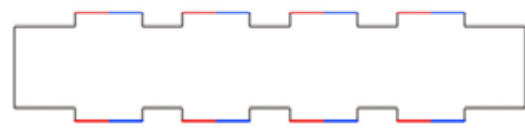

$\mathrm{A}_{2}$

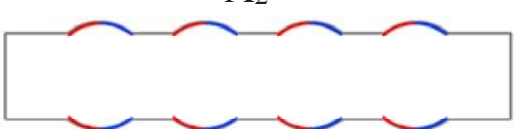

$\mathrm{B}_{2}$

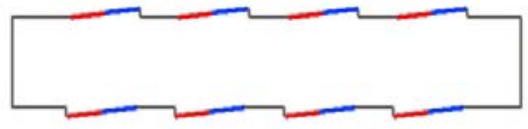

$\mathrm{C}_{2}$

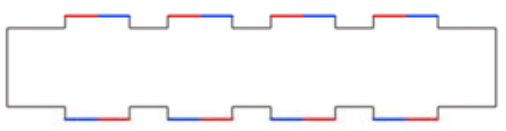

$\mathrm{A}_{3}$

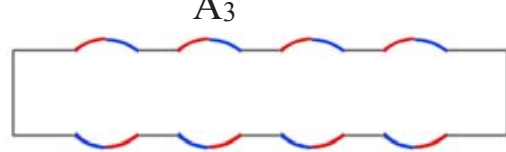

$\mathrm{B}_{3}$

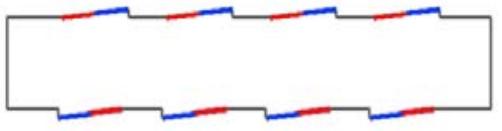

$\mathrm{C}_{3}$

Fig.1 Different zeta potential distribution in three channel structures

The size of entrance and exit, inner space of the channel, amount of mixing elements will be the same. Flow rate, concentration, and diffusivity are set identically to make the results more comparable.

\subsection{Parameter settings}

Tab 1 Initialization parameters design

\begin{tabular}{ccc}
\hline Parameter & value & item \\
\hline $\boldsymbol{\rho}$ & $1000 \mathrm{~kg} / \mathrm{m}^{3}$ & Density \\
$\boldsymbol{\eta}[12-15]$ & $10^{-3} \mathrm{~Pa} \cdot \mathrm{s}$ & Liquid viscosity \\
$\mathbf{U}[11-16]$ & $1 \mathrm{~mm} / \mathrm{s}$ & Average speed in the Entrance \\
$\boldsymbol{\varepsilon}_{\mathbf{r}}$ & 80.2 & Dielectric constant \\
$\boldsymbol{\zeta}$ & $\pm 0.25 \mathrm{~V}$ & Zeta potential value \\
$\boldsymbol{\sigma}$ & $0.11845 \mathrm{~S} / \mathrm{m}$ & Electrolyte solution perveance \\
$\mathbf{D}[11-16]$ & $10^{-11} \mathrm{~m}^{2} / \mathrm{s}$ & Diffusion coefficient \\
$\mathbf{C}[12-14]$ & $1 \mathrm{~mol}^{3} / \mathrm{m}^{3}$ & Initial solution concentration \\
$\mathbf{L}[11,13]$ & $10^{-4} \mu \mathrm{m}^{2}$ & Length of Entrance \\
$\mathbf{S}[11,13]$ & $737.5 \mu \mathrm{m}^{2}$ & Scale of Channel \\
\hline
\end{tabular}

\section{Mathematical Modeling}

\subsection{Electroosmotic Flow}

Electroosmotic flow in micro scale is viscous flow under low Reynolds number and is incompressible. The equation for boundary conditions is [17],

$$
\begin{aligned}
& \rho\left[\frac{\partial \boldsymbol{u}}{\partial t}+(\boldsymbol{u} \cdot \nabla) \boldsymbol{u}\right]+\nabla P-\mu \nabla^{2} u=0 \\
& \nabla \cdot \boldsymbol{u}=0
\end{aligned}
$$

In (1), $\boldsymbol{u}$ represents flow velocity, $\mu$ represents viscosity, P represents pressure. Dirichlet boundary condition is applied including inlet as velocity boundary, exit as pressure boundary, the rest are wall boundary. For the wall with double electric layer. The equation for boundary conditions is[18],

$$
\boldsymbol{u}=\frac{\varepsilon_{w} \zeta_{0}}{\mu} \nabla V
$$


In (3), $\varepsilon_{w}$ represents dielectric constant, $\varepsilon_{w}=\varepsilon_{0} \varepsilon_{r}, \varepsilon_{0}$ represents vacuum permittivity, $\varepsilon_{r}$ represents relative dielectric constant. $\zeta_{0}$ represents zeta potential, $\nabla V$ represents potential gradient.

\subsection{Static Field}

Electric power distribution can be defined under Ome law and current density formula [18]:

$\nabla \cdot(-\sigma \nabla V)=0$

$\sigma$ represents conductivity, part in brackets represents current density.

\subsection{Convective Diffusion}

Without chemical reactions, the movements mainly include transportation and diffusion [19].

$$
\frac{\partial c}{\partial t}+\nabla \cdot(-D \nabla c)=-\mathbf{u} \cdot \nabla c
$$

In (5) c represents concentration, $D$ represents diffusion coefficient.

\subsection{Definition of Mixing Efficiency}

In the exit of the channel, mixing efficiency was defined as [20]:

$\left[1-\frac{\int_{0}^{l}\left|c-c_{\infty}\right| d l}{\int_{0}^{l}\left|c_{0}-c_{\infty}\right| d l}\right] \times 100 \%$

In (6), $c$ represents original concentration, $c_{\infty}$ represents concentration after mixture, $c 0$ represents concentration before mixture, $l$ represents the width of exit.

\section{Results of simulation}

\subsection{Passive Mixing}

When electroosmotic flow is under default setting, vortex cannot be effectively formed in the channel to disturb the solute diffusion. Solute is mixing mainly by diffusion and the condition is showed in the diagram 2 (a).

The diagram shows that despite the different structure of two walls, the performance and distribution are similar with efficiency both reaching 50\%, which shows in micro scale, changing the form of wall cannot improve the mix efficiency.

\subsection{Active mixing}

With different types of wall and Zeta potential distribution in the channel, electroosmotic flow can be formed by adjusting electricity voltage thus producing vortex by changing the flow condition. Zeta potential value are $0.25 \mathrm{~V}$ and $-0.25 \mathrm{~V}$ (as defined in 2.2) respectively, difference between the entrance and exit will increase from $0.1 \mathrm{~V}$ to $0.5 \mathrm{~V}$. The mix efficiency will be tested under different voltage.

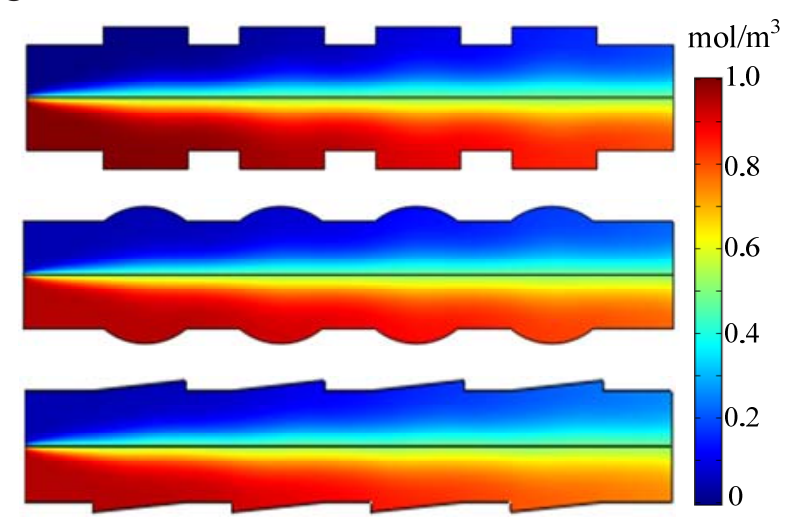

(a) concentration distribution

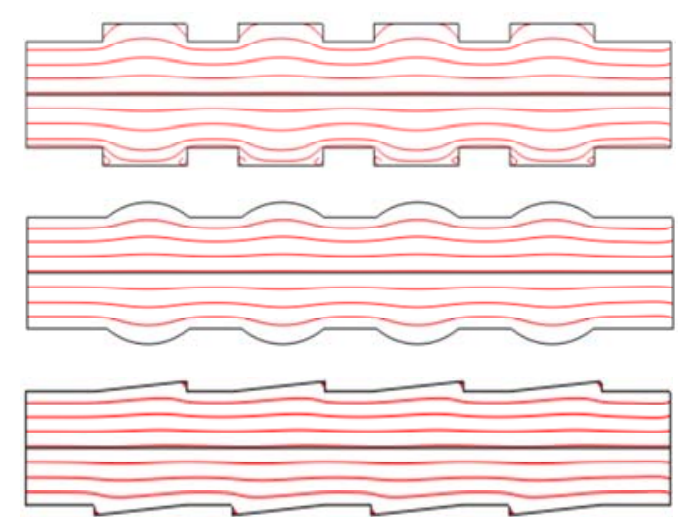

(b) fluid flow distribution

Fig 2. Concentration distribution and fluid flow distribution in passive micromixer

The diagram below shows the voltage difference between the entrance and exit is $0.5 \mathrm{~V}$. Using the Zeta potential distribution in Type $\mathrm{C}$, solute diffusion condition and fluid flow distribution are showed in the diagram below. Due to the difference of wall types and zeta potential distribution, vortex is different. In comparison, the slope and arc type can make it form larger vortex. Fluid flow 
through are fully folded, which means electroosmotic flow has a great influence on the flow in the channel. The results in rectangle type are opposite. Therefore, the mixing efficiency in slope and arc are better than rectangular shape. 3(a) shows the concentration distribution in the process of flow. Compared with 2(a), concentration in the exit is close to the average, which means vortex produced by electroosmotic flow can effectively improve the mixing efficiency. In addition, in terms of three mixing conditions of exit in 3(a), the same conclusion can be made with the fluid flow distribution analysis-the mixing efficiency in slope and arc are better than rectangular shape.

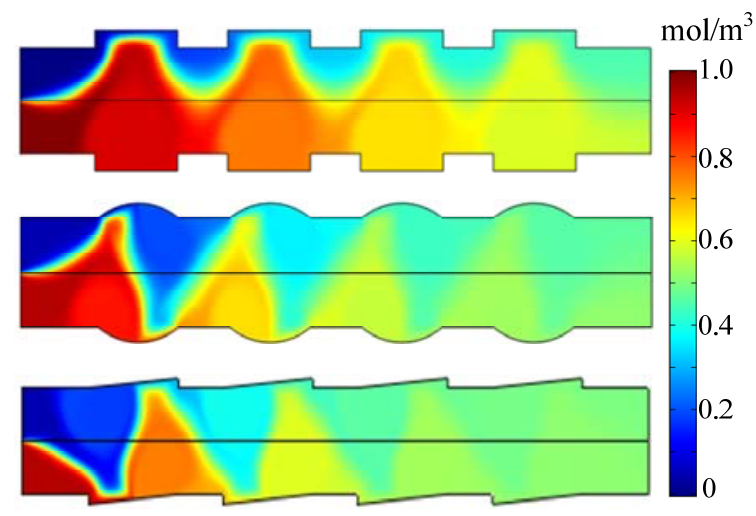

(a) concentration distribution

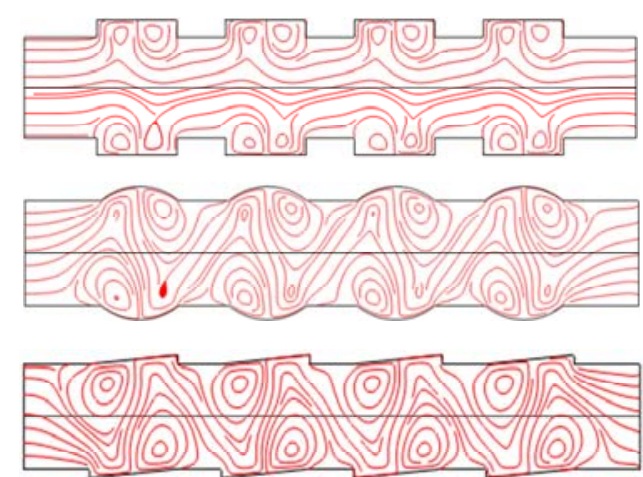

(b) fluid flow distribution

Fig 3. Concentration distribution and fluid flow distribution of samples in active micromixer

\section{Characteristics of Mixing}

To understand the influence of driving voltage and zeta potential distribution on the mix efficiency. Above nine designs are compared with setting the voltage difference from $0.1 \mathrm{~V}$ to $0.5 \mathrm{~V}$. The efficiency of model A is showed below. A1's mix efficiency was remarkably improved with the increase of driving voltage. When the driving voltage reached $0.5 \mathrm{~V}$, the mix efficiency reached $90.6 \%$. As for A2 and A3 ,the efficiency has not been improved much, only reaching $60 \%$.

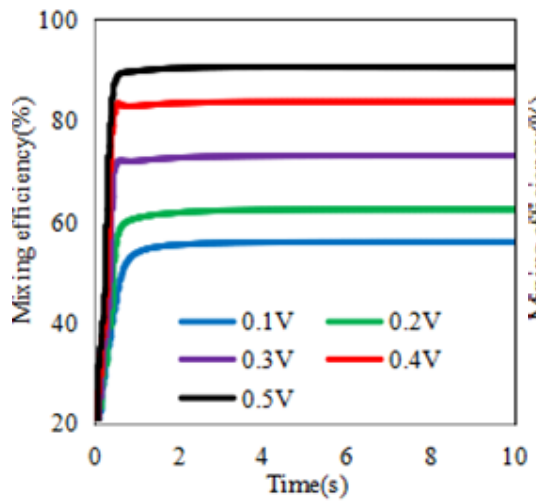

$\mathrm{A}_{1}$

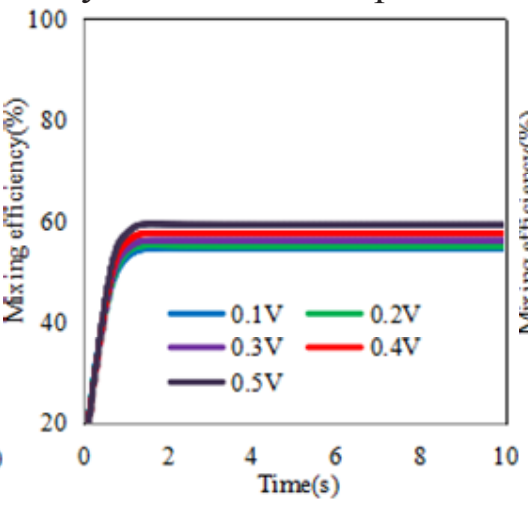

$\mathrm{A}_{2}$

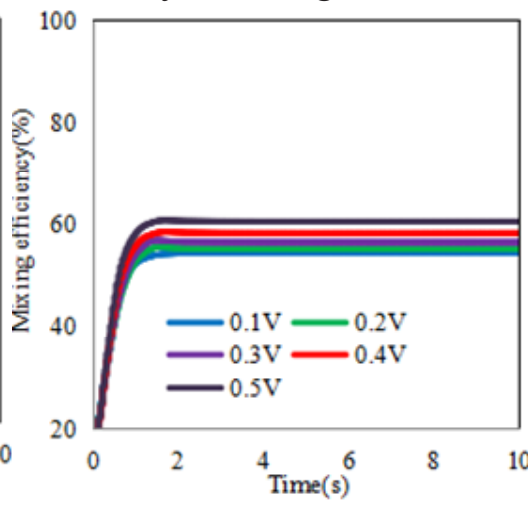

$\mathrm{A}_{3}$

Fig 4. Mixing characteristics of three kinds of designs under different voltage in model A

As is showed in the diagram below, although, amount of vortex produced in A1 is obviously less than A2 and A3, the range of A1 is larger than A2's and A3's. Therefore, the scale of the vertex is the key factor that influence the mix efficiency.
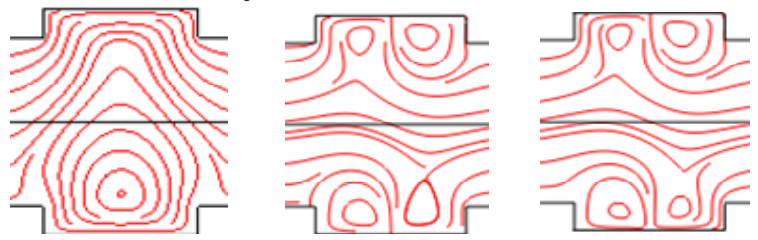

Fig 5. Fluid flow distribution with three kinds of potential Settings in model A

The diagram 6 illustrate the characteristic of B types. Under 0.5 driving voltage, B3 can get most efficient mixing, which is $97.6 \%$, followed by B2 with $81.5 \%$. Under 0.3 driving voltage, B3's mix 
efficiency can reach $89.1 \%$, while B3's is $75.6 \%$. The result is same as it under $0.2 \mathrm{~V}$ or $0.1 \mathrm{~V}$. Accordingly, under low driving voltage, B1 has an advantage against B3.

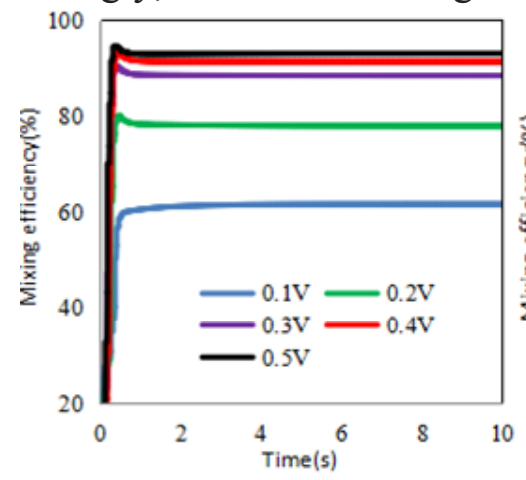

$\mathrm{B}_{1}$

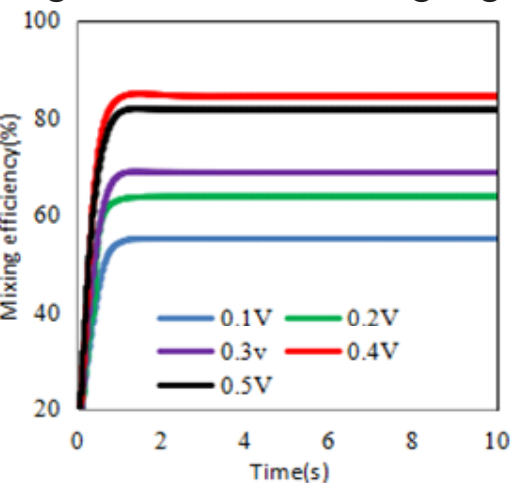

$\mathrm{B}_{2}$

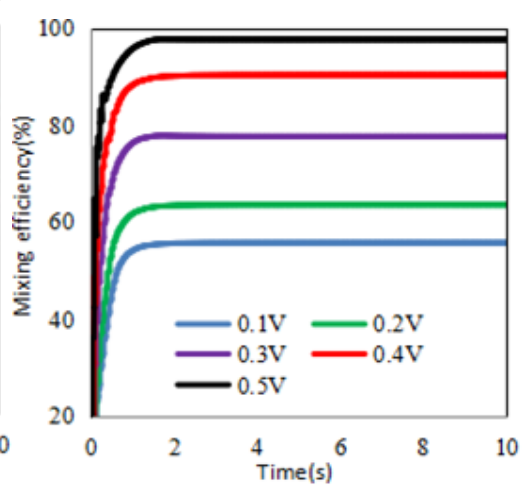

$\mathrm{B} 3$

Fig 6. Mixing characteristics of three kinds of schemes under different voltage in model B

The mixing characteristics of type $\mathrm{C}$ can be overserved in the diagram below. The mix efficiency of all the three models in $\mathrm{C}$ type can reach more than 95\%. Overall, C3 is the most efficient with 97.5\%. Additionally, $\mathrm{C} 1$ and $\mathrm{C} 2$ both show an efficient mixing process under low driving voltage, which is better than B1.
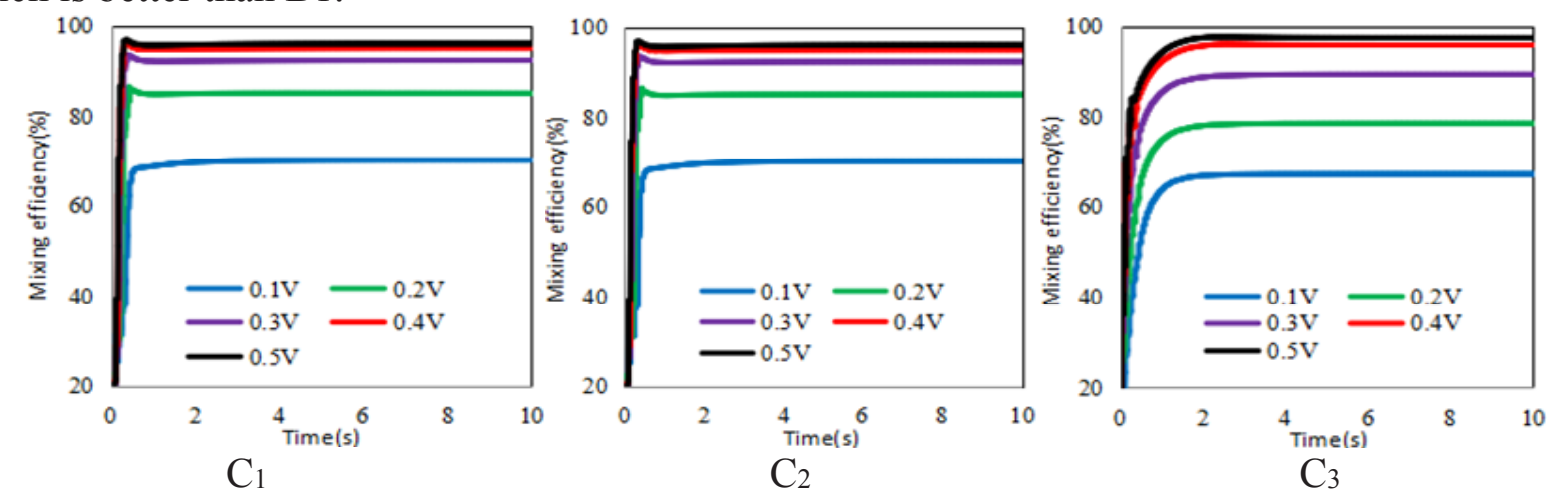

Fig 7. Mixing characteristics of three kinds of designs under different voltage in model $\mathrm{C}$

Base on the research above, different types of wall and zeta potential distribution will lead to different mixing efficiency under the same scales of channel, size of the entrance and exit, diffusion coefficient. Overall, the mixing process of $\mathrm{C}$ model is most efficient. The mixing efficiency of all three models are kept in a stable condition after electrifying for 2 seconds, which means direct electroosmotic flow are more stable than alternating current[11].

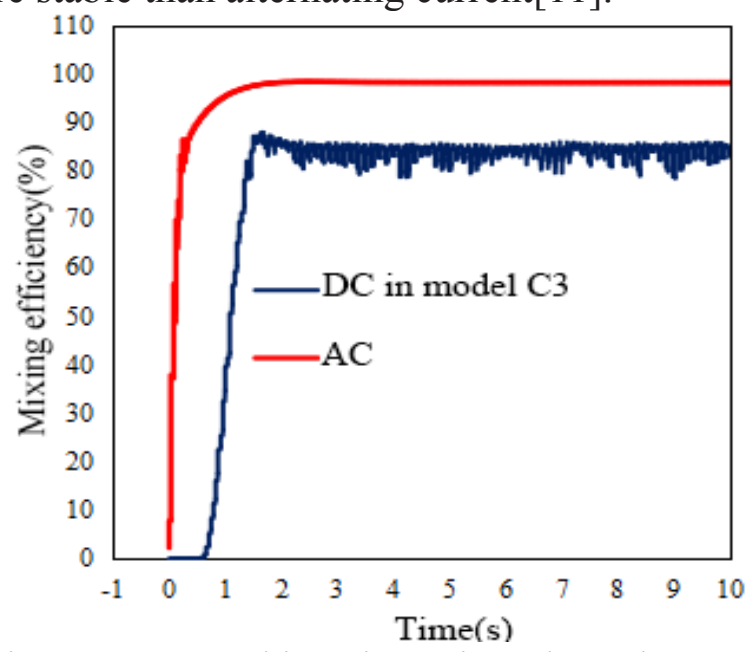

Fig 8. Mixing efficiency compared in Micromixer drove by DC in $\mathrm{C} 3$ and $\mathrm{AC}[11]$ 


\section{Conclusion}

Nine electroosmotic micromixers based on direct current are designed with different types of wall and Zeta potential distribution. In conclusion, under the same scales of channel, size of the entrance and exit, diffusion coefficient, the mixing efficiency is totally different by changing the wall types and Zeta potential distribution. Moreover, vortex produced near the interface of solute concentration a key factor to consider in improving the efficiency. Electroosmotic micromixer under the direct current are more stable than under the alternating current.

\section{Acknowledgements}

This work is supported by the National Natural Science Foundation of China (No.81301291 and No. 81471743).

\section{References}

[1] Kutter, J. P. Trends Anal. Chem. "Current developments in electrophoretic and chromatographic separation methods on microfabricated devices,"Trac Trends in Analytical Chemistry,2000, 19, 352-363.

[2] N T. Nguyen, "Micromixers Fundamentals, Design and Fabrication," William Andrew, Elsevier, 2nd ed, 2012.

[3] D. Gobby, P. Angeli and A. Gavriilidis, "Mixing characteristics of T-type microfluidic mixers," Journal of Micromechanics and Microengineering (11) (2001) 126-132.

[4] M. Yi and H. H. Bau, "The kinematics of bend-induced stirring in micro-conduits, Proc. ASME Intl," Mechanical Engineering Congress and Exposition, MEMS 2 (2000) 489-496.

[5] V. Studer, A. Pepin, Y. Chen and A. "An integrated AC electrokinetic pump in a microfluidic loop for fast and tunable flow control.Ajdari," Analyst, 2004, 129,944.

[6] S. Debesset, C. J. Hayden, C. Dalton, J. C. T. Eijkel and A. "An AC electroosmotic micropump for circular chromatographic applications," Manz, Lab Chip, 2004, 4, 396.

[7] H. Chen, Y.T. Zhang, I. Mezic, C.D. Meinhart, and L. Petzold, "Numerical simulation of an electroosmotic micromixer," Proc Microfluidics 2003 (ASME IMECE), 2003.

[8] Teng Zhou, Hanlin Wang, Liuyong Shi, Zhenyu Liu, and Sang Woo Joo, "n Enhanced Electroosmotic Micromixer with an Efficient Asymmetric Lateral Structure," Micromachines, 2016, 7, 218.

[9] Mohammad Maadi, and Tuhid Pashaee Golmarz, "Investigation of mixing and simulation of an electroosmotic micromixer,"Journal of Mechanical Science and Technology, 28 (8) (2014) 3223 3230.

[10] Shahrzad Forouzanfar, Nima Talebzadeh,Siavash Zaegri, Hadi Veladi," The Effect of Microchannel width on Mixing Efficiency of Microfluidic Electroosmotic Mixer,"Rsi International Conference on Robotics \& Mechatronics, 2015.

[11]Mersal, G. A. M., Bilitewski, U., "Manipulation of the electroosmotic flow in glass und PMMA microchips with respect to specific enzymatic glucose determinations," Microchim Acta 2005, 151, 29-38.

[12] Yanting Zhang, Igor Mezic, Carl Meinhart,Linda Petzold, "Numerical simulation of an electroosmotic micromixer" November 15-21, 2003:1-6.

[13]H Hadidi, R Kamali, "Numerical simulation of a non-equilibrium electrokinetic micro/nano fluidic mixer," Journal of Micromechanics and Microengineering, 26 (2016) 035019 (8pp).

[14]S.R. Karumuri, P. Nirmala, M. Krishnajee, et al, "Simulation of Electro Osmotic Micro Mixer in Micro-Electro-Mechanical-System: An Application to Bio-medical Field," IJASETR 1(1): Article.

[15]Teng Zhou, Hanlin Wang, Liuyong Shi, Zhenyu Liu, and Sang Woo Joo 2, "An Enhanced Electroosmotic Micromixer with an Efficient Asymmetric Lateral Structure," micromachines, 1 December 2016. 
[16]M Maadi, TP Golmarz,"Investigation of mixing and simulation of an electroosmotic micromixer,"Journal of Mechanical Science and Technology, 2014, 28(8): 3223-3230.

[17]Zhang Yubao, Li Qiang, "Modeling and application of MEMS based oncomsol multiphysics," Beijing: Metallurgical Industry Press, 2007: 159-160.

[18]Chen H, Zhang Y T, Mezic I, et al. "Numerical simulation of anelectroosmotic micromixer ,"Proceedings of Microfluidics 2003 ASMEIMECE. Washington DC, 2003.

[19]Ermakov S V, Jacobson S C, Ramsey J M." Computer simulation ofelectrokinetic injection techniques in microfluidic devices," AnalyticalChemistry, 2000, 72: 3512-3517.

[20]C C. Cho, "Electrokinetically driven flow mixing utilizing chaoticelectric fields," Microfluidics and nanofluidics 5, no. 6, pp. 785-793, 2008. 